\section{$\underset{\text { hommes }}{\text { \& migrations }}$}

\section{Hommes \& migrations}

Revue française de référence sur les dynamiques

migratoires

$1281 \mid 2009$

France-Brésil sous l'angle des migrations et de l'altérité

\title{
Sous le signe du soleil
}

\section{Mustapha Harzoune}

\section{Q OpenEdition}

1 Journals

\section{Édition électronique}

URL : http://journals.openedition.org/hommesmigrations/412

DOI : 10.4000/hommesmigrations.412

ISSN : 2262-3353

\section{Éditeur}

Musée national de l'histoire de l'immigration

\section{Édition imprimée}

Date de publication : 1 septembre 2009

Pagination : 196-203

ISSN : 1142-852X

\section{Référence électronique}

Mustapha Harzoune, « Sous le signe du soleil », Hommes \& migrations [En ligne], 1281 | 2009, mis en ligne le 29 mai 2013, consulté le 22 septembre 2020. URL : http://journals.openedition.org/ hommesmigrations/412 ; DOI : https://doi.org/10.4000/hommesmigrations.412

Ce document a été généré automatiquement le 22 septembre 2020.

Tous droits réservés 


\title{
Sous le signe du soleil
}

\author{
Mustapha Harzoune
}

1 Ainsi, les "expulsables" ont été expulsés, et à tour de bras encore, à en croire les communiqués des associations RESF ou l'Anafé. On a même vu une agence du ci-devant Crédit Lyonnais transformée en traquenard pour un certain Mamadou, gratifié, pour le coup, de plusieurs jours d'enfermement (L'Humanité du 14 août)! D'ailleurs, celles et ceux qui, selon la technocratique formule, avaient "vocation" à être retenus enfermés ont bien été internés et, parmi eux, des enfants (voir le "débat autour de la rétention des enfants", publié le 13 août sur le site de L'Express). Dans une dépêche, l'AFP rapportait le 4 août que la Cimade avait demandé

"la remise en liberté de plusieurs familles placées en rétention avec leurs enfants [...]. Selon la Cimade, une fillette de quatre ans et ses deux parents, présents en France depuis plusieurs années, sont retenus depuis le 30 juillet au CRA de Marseille et peuvent être reconduits 'd'un jour à l'autre' vers les Philippines.

Une autre famille, avec un enfant âgé de huit ans, est maintenue depuis fin juillet au CRA de Metz, selon l'association, qui affirme que son renvoi vers l'Albanie est 'imminent'.

Une mère et sa fille de deux ans ont quant à elles été placées au CRA de Toulouse et sont susceptibles d'être renvoyées au Togo, alors que le père a été libéré par une décision de justice [...]".

$2 \mathrm{Si}$, pour Éric Besson, le délit de solidarité est une fable inventée par quelques démagos, il a fini par convenir - sans convaincre les associations - de la nécessité de clarifier la loi (voir Libération du 17 juillet et Le Monde du 18). Il faut dire que deux affaires ont tout de même affaibli les dires du ministre : l'affaire Salimon Fofana à Rodez (voir Libération du 24 juin et Le Nouvel-Obs.com du même jour) et l'affaire Claudine Louis du côté de Saint-Girons (voir La Dépêche du 22 juillet). Ainsi, si l'on n'est pas forcément condamné pour délit de solidarité, il semble bien que délit il y ait, et que, de ce fait, les ennuis puissent tomber sur la tête du premier citoyen entiché d'humanisme ou d'humanité. 


\section{Délit de sale gueule!}

3 Tandis que la commission sur le port de la burqa auditionnait et s'interrogeait sur le mystère de ces visages qui se soustraient au regard de leurs semblables, pour d'autres, circuler dans l'espace public à visage découvert revenait à s'exposer au délit de sale gueule ou de faciès (voir la tribune intitulée "Le retour sournois du délit de faciès" signée par William Bourdon, avocat et président de l'association de juristes Sherpa, Le Monde du 18 juillet). Ali Ziri, frêle retraité de 69 ans, pourrait bien en avoir été la malheureuse victime. Il était en voiture avec un ami lorsqu'il a été contrôlé par la police du côté d'Argenteuil. Contrôle musclé, semble-t-il, puisque les deux compères auraient été violentés, et Ali Ziri est mort! Un collectif pour demander l'ouverture d'une enquête s'est monté et l'écrivain Mouloud Akkouche a mis en balance le déséquilibre entre les droits exorbitants accordés à la police et l'impuissance du citoyen lambda à simplement faire valoir les siens : "Des policiers frappent un retraité, il meurt. Qui s'en soucie? interroge$t$-il (Rue89, le 19 juillet).

4 À propos d'un tout autre délit de faciès, retraçant la bio de la journaliste russe et militante de l'ONG Mémorial en Tchétchénie, Natalia Estemirova, assassinée le 15 juillet, Le Monde rapporte cet épisode qui opposa le 31 mars 2008 la journaliste au président Ramzan Kadirov. Convoquée par ce dernier, le quotidien écrit :

“Natalia ne porte pas le foulard désormais imposé aux femmes. 'Tu dois te comporter comme une femme respectable, pas comme une pute', lui aurait dit 'le tout-puissant président tchétchène'. 'Tu me provoques, tu m'excites avec tes cheveux', crie-t-il entre deux bordées de jurons."

5 À Oullins dans le Rhône, Fatima Z, 21 ans, ne portait pas le voile non plus. Elle a été tuée dans une cave.

"Le frère de la victime, Mohammed, 17 ans, a été mis en examen pour homicide volontaire et écroué jeudi. Il est accusé d'avoir étranglé Fatima avant de brûler son corps. Muré dans le silence depuis son arrestation, le jeune garçon nie les faits." (Libération, 10 juillet)

"Jugeait-il la vie de sa sœur trop légère ?" interroge Le Monde (12 juillet).

Samira, la meilleure amie de la victime, fulmine :

"J'ai la haine, on se fait frapper parce qu'on est des femmes, c'est un quartier pourri." (Libération, 10 juillet) Pour Le Monde, "dans le quartier l'hypothèse d'un frère qui aurait voulu punir une sœur à la vie trop légère à son goût revient le plus souvent".

7 Laconique, le journaliste Richard Schittly écrit: "Interprétation délicate, incertaine". Puis il donne la parole au père de la victime qui dénonce, à propos de son fils, "des fréquentations extérieures, dans un quartier où règne 'l'omerta', qui auraient pu lui ' remonter la tête"'.

\section{Banlieue : les mots pour le dire...}

8 L'Insee, lui, "cacherait" un certain fichier "Saphir", ce qui, pour le chroniqueur du Figaro Ivan Rioufol, s'apparenterait à un "mensonge d'État". Michèle Tribalat et Bernard Aubry ont travaillé sur les données dudit fichier. Leur article a été publié dans le numéro de juin de la revue Commentaire. Voici comment le numéro de Marianne du 22 juillet résume ce travail : 
“37\% de jeunes d'origine étrangère en Île-de-France, plus de $60 \%$ dans une vingtaine de villes, explosion du nombre de jeunes originaires d'Afrique subsaharienne, proportion de jeunes d'origine étrangère en très forte hausse dans l'ouest de la France..." quelques chiffres, ne mériterait-elle pas une analyse plus sérieuse? Qu'importe! Cet été, la banlieue a encore fait parler d'elle. À Bagnolet surtout, après la mort de Yakou Sanogo, dix-huit ans, survenue après "une poursuite avec la police" (L'Humanité, 11 août).

Pour Marc Everbecq, le maire communiste de Bagnolet,

"la tension règne, depuis plus d'un an dans toutes les villes populaires. Les jeunes entretiennent des rapports difficiles avec la police, mais aussi avec leurs ainés, avec les institutions républicaines, avec leurs familles." Il parle ainsi d'"une nette dégradation sociale": "Les dispositifs se succèdent, se superposent, sans réelle efficacité. Privés de parole, privés d'avenir, les jeunes des quartiers populaires ont l'impression de hurler dans le désert. L'exaspération s'exprime de tous les côtés, alimentant un cercle vicieux de méfiance mutuelle."

11 "Les jeunes des quartiers populaires ont l'impression de hurler dans le désert", dit Marc Everbecq, tandis que le linguiste Alain Bentolila dans le quotidien Libération du 24 juillet montrait le cheminement qui va "de l'impuissance linguistique à la violence". "Si nos enfants - je dis bien nos enfants - passent à l'acte plus vite et plus fort, c'est parce que ni nous ni leurs maîtres n'avons su leur transmettre la capacité de mettre pacifiquement en mot leur pensée pour l'Autre." Et d'ajouter : "Leur drame est que l'école n'a pas su leur donner le goût de l'Autre." Il faut lire la description faite par le linguiste d'une journée passée dans un tribunal d'instance. Édifiant! Alain Bentolila décrit " une parodie de justice sur fond d'illettrisme" et "l'enchaînement fatal entre impuissance linguistique et passage à l'acte violent". Non seulement les jeunes des quartiers hurlent dans le désert, mais ils ne trouvent même pas les mots pour le faire!

\section{Vous avez dit "Eldorado" ?}

Enfin, malgré les difficultés qui s'amoncellent au-dessus de la tête des sans-papiers, on continue à servir la farce de l'Eldorado. À Calais, alors que la gale faisait son apparition, un accord franco-britannique laisse à penser, du moins du côté du MRAP, que des charters pourraient être remis au goût du jour. Quoi qu'il en soit, pour Le Monde (8 juillet), l'arrangement entre la France et la Grande-Bretagne à propos de la question calaisienne conclut à Evian "ne pouvait se faire sans mesures répressives fortes. Ainsi les deux parties sont convenues d' 'augmenter de manière significative le nombre de retours forcés d'étrangers en situation irrégulière et leur réintégration dans leurs pays d'origine ou de transit ". Un autre indicateur montre que le soleil noir de l'exil s'assombrit: "Les envois de fonds des émigrés à leurs familles devraient baisser de 7,3\% en 2009, à 304 milliards de dollars (218 milliards d'euros), selon une étude de la Banque mondiale." (Le Monde du 15 juillet)

Dans l'Eldorado mythique, l'or devait se ramasser à la pelle. Les immigrés, clandestins ou pas, n'ont rien de conquistadors, et ce qu'ils ramassent, dans le meilleur des cas, ce ne sont que quelques subsides, payées souvent au prix fort ! 


\section{Avignon, le temps des vacances}

14 Avignon est le rendez-vous de l'été. L'artiste associé de la $63^{\mathrm{e}}$ édition du festival était le libano-québécois Wajdi Mouawad. Relatant les quelque onze heures de représentation de ses trois pièces - Littoral, Incendies et Forêts - devant près de 2000 spectateurs, l'envoyée spéciale du Monde écrit :

“Presque personne n'a déserté la Cour d'honneur, où il y avait beaucoup de jeunes gens, attirés par un bouche-à-oreille qui fait de Wajdi Mouawad un des rares créateurs aptes à donner le goût du théâtre aux nouvelles générations, et à élargir le cercle du public à ceux qui d'ordinaire ne viendraient pas passer une nuit au palais des papes."

Mais Avignon 2009 fut aussi l'occasion d'apprécier d'autres artistes qui tirent la culture nationale vers le haut - pour paraphraser le personnage de Sonia dans le dernier Pierre Assouline.

Ainsi, le chorégraphe Rachid Ouramdane y a présenté Des témoins ordinaires, une mise en scène des "guerres, tortures et génocides", "des souffrances endurées lors de guerres civiles" (L'Humanité du 23 juillet).

Du côté du off, D'de Kabal a donné son spectacle, Écorce de peine, dans lequel il met en mots et en mouvements l'histoire de l'esclavage et l'histoire d'un gamin qui a grandi de l'autre côté du périph. À propos du off, Frédéric Martel consacrait son émission - Masse critique sur France Culture, le 18 juillet - à un thème unique :

"La multiplication des spectacles arabo-berbères, ou maghrébins ou encore des spectacles beurs. Les acteurs arabes, les textes écrits par des Français issus de l'immigration, les producteurs français d'origine maghrébine sont nombreux cette année dans le off. De plus en plus nombreux. Pourquoi ? Est-ce parce que le in ne les accueille pas assez, en dépit de Wajdi Mouawad [...], est-ce parce que le public des quartiers qui fuit le in serait un peu plus disposé à se rendre dans le off? Est-ce parce que cibler les Beurs est un bon marketing? Qu'en est-il enfin de notre sacrosainte diversité culturelle proclamée dans les enceintes de l'OMC et de l'Unesco mais peu appliquée sur le terrain ici à Avignon?"

Parmi les invités, il y avait Faïza Kaddour, qui jouait Le Frichti de Fatou, un texte sur l'identité d'une femme algérienne, l'exil, les violences conjugales, mais aussi sur l'émancipation et la sexualité. "Ce spectacle est un hommage à mon père. Je m'appelle Faïza Kaddour, mais je ne parle pas la langue de mon père. J'ai voulu aussi lui rendre un petit hommage par cette identité que je n'ai pas, mais que j'ai par ma famille..." L'animateur lui demande alors si elle cherchait à "avoir un public qui vienne des quartiers, car, sans critique aucune, la salle était plutôt 'blanc caucasien', comme on dit aux États-Unis". Il poursuit : "Vous n'avez pas cherché spécifiquement à parler à la communauté franco-maghrébine? Cela ne fait-il pas partie d'un objectif?" Et Faïza Kaddour de répondre : "Quand on nous le demande, on va voir parfois des personnes qui n'ont pas l'habitude d'aller au théâtre [...]. Mais, non, pas spécialement." Point à la ligne donc.

19 Farid Omri donnait lui à Avignon le Couscous aux lardons. Une façon de traiter sur le mode comique de sujets sérieux et parfois graves :

"Ce n'était pas une volonté didactique ou militante... c'était juste [pour] rire ensemble de nos travers et de nos différences, ce qui aujourd'hui est devenu malheureusement très très difficile. Toutes les identités sont très exacerbées, les gens sont très farouches." 
Couscous aux lardons raconte l'histoire d'un couple mixte, qui se questionne - différence culturelle oblige - sur le prénom à donner au fiston qui arrive. Intervention du journaliste :

"Parlons business. Au fond, vous jouez la carte maghrébine parce que cela rapporte à Avignon?"

Et Farid Omri de plaisanter :

"Oui, oui, j'hésitais entre le théâtre et l'épicerie, et finalement j'ai choisi le théâtre."

\section{"Le théâtre du coin " à l'instar de "l'Arabe du coin "...}

Ensuite, ce fut au tour de Michel Gazi, directeur de l'espace social et culturel de la Croix des oiseaux, un des quartiers sud d'Avignon, de répondre. À propos des habitants de ces quartiers, il explique :

"Il y a peu de différence entre le in et le off pour eux : ils n'y vont pas beaucoup, ça c'est certain. C'est en train de changer. Il y a des artistes qui vont au-devant des habitants des quartiers. Il y a des animateurs, des travailleurs sociaux qui font en sorte que la culture leur soit accessible. Ce n'est pas une question d'argent simplement, il y a aussi et simplement un rapport à la culture qui est différent. Il y a le problème de l'interculturalité aussi, il y a beaucoup de nationalités différentes ; il y a le rapport à la culture française qui n'est pas toujours simple... Tout cela fait que quand il y a plus de 1000 pièces de théâtre dans le off et quelques-unes dans le in, il y en a trop. On ne sait pas où donner de la tête..."

Et sur l'insistance du journaliste, à propos du caractère "isole" du festival, il ajoute :

“Je ne pense pas qu'on règle le problème en faisant des spectacles dit 'spécialisés' pour un public 'spécialisé'... Qu'il y ait des passerelles, des moyens pour accompagner les personnes, oui, mais les artistes n'ont pas à s'adapter à cela, n'ont pas à modifier leur façon d'être, d'écrire, de créer parce qu'il y a une difficulté quelconque [...]."

D'ailleurs, Armelle Elio, critique théâtre au Figaro, notait :

"Moi, je suis frappée par l'évolution des spectateurs dans les salles, aussi bien du in que du off. Évidemment, c'est encore plus frappant dans le off, il y a beaucoup de jeunes et beaucoup de jeunes dont on se dit qu'ils sont issus de la troisième ou de la quatrième génération du Maghreb ou d'autres pays."

L'émission évoqua aussi la trilogie algérienne de Rachid Akbal, qui raconte la mémoire de la mère et de la Terre-mère, parle d'identité et d'immigration, de la guerre d'Algérie...

"Moi, je revendique une écriture qui n'est pas maghrébine en soi et communautariste. C'est un choix d'avoir choisi une langue même quelquefois très littéraire pour au contraire donner un souffle à ces passages que je voulais très épiques, très forts, où on parle de la souffrance de l'immigré ou du moment du choix de l'exil. Je voulais utiliser ici une autre langue que celle du quotidien. Par contre, j'utilise la langue du quotidien, la langue des cafés pour parler de la guerre. "

À Avignon, il était aussi possible de visiter l'exposition-dénonciation de Jean Michel Bruyères sur les centres d'accueil administratifs des immigrés ou d'assister à Sacrifices, le troisième spectacle de Nouara Naghouche, dans lequel elle évoque les mariages forcés, l'enfermement de certaines femmes par leur conjoint ou le machisme. 


\section{“Agir avec les mots"}

La culture était aussi du côté de Tours, grâce à Kader Attia qui y présentait Kasbah. Une exposition d'environ $350 \mathrm{~m}^{2}$ qui

“incarne la réflexion que Kader Attia mène depuis une dizaine d'années sur l'état $\mathrm{du}$ monde, la réalité sociale, les fractures Nord-Sud, Occident-Orient, la pauvreté, le déracinement... et le thème de la réappropriation culturelle toujours au centre de la démarche de l'artiste d'origine algérienne, né en 1970 à Dugny, en Seine-SaintDenis et aujourd'hui installé à Berlin " (Libération, 25-26 juillet).

De son côté, Le Monde du 10 juillet brossait le portrait de Larsen, un rappeur "méconnu du grand public", mais qui serait

"une des rares personnes à pouvoir rencontrer tous les gamins des cités de l'île-deFrance". "Hacène Souadji, connu sous son nom de scène, Larsen, 26 ans, a grandi à Tremblay-en-France, en Seine-Saint-Denis, et a l'apparence du rappeur des basfonds, spécialiste dans l'art de la provocation et de la rime en 'nique'. Larsen n'est pas Diam's ou NTM, mais avec son dernier album Du seum bien vicère - expression à double sens qu'on peut traduire par 'du cannabis bien servi' ou 'de la vraie rage' -, il en est à son neuvième disque."

L'article évoque ensuite les années de prison :

"La visite de son premier enfant au parloir l'a bousculé. La découverte de l'islam qu'il ne pratiquait pas - lui a donné un cadre."

Tiens! Hier, seuls les vieillards renouaient avec les pratiques religieuses, histoire de se racheter une conduite avant de passer au "trébuchet de l'âme" ; aujourd'hui, ce sont des jeunots qui s'échinent à redorer un blason trop tôt terni... Enfin, le rap chez Larsen est devenu un espace d'expression. "J'ai usé les armes de la violence. Puis, j'ai fini par comprendre qu'il fallait agir avec les mots." Voilà qui rappelle les propos d'Alain Bentolila.

\section{Quand l'été fait son cinoche}

2 L'écran noir reste le grand pourvoyeur de célébrités. De ce côté aussi, la période estivale méritait d'abandonner, le temps d'une projection, les bains de mer et de soleil.

3 Abdel Raouf Dafri est devenu “à 45 ans, le nouveau Wünderkid du cinéma français : scénariste de La Commune, du Mesrine de Jean-François Richet, et inspirateur d'Un Prophète de Jacques Audiard", un film qui a révélé un nouveau talent en la personne de Tahar Rahim (Les Inrocks.com du 25 août).

34 Abdel Raouf Dafri est sans doute "une grande gueule" :

"Je veux pouvoir me regarder dans la glace, plutôt que de persister et de me faire entuber. Je ne me renie pas. [J'ai été] élevé dans les quartiers défavorisés de Wattignies, près de Lille [...], mes parents sont analphabètes. Je me suis fait dégager du système scolaire, et j'ai atterri en CAP. À l'époque, c'est ce qu'on faisait pour la plupart des Arabes."

35

Et Dafri de poursuivre : “Ce qui m'a sauvé, c'est de lire. Je lisais tout. Et puis comme je n'avais pas d'argent, je lisais les bouquins au supermarché. J'ai découvert Balzac, Zola..." Devenu un temps animateur télé, Abdel Raouf Dafri se fait appeler "Francis Panama". "Et ça passe malgré l'absurdité de la situation", précise-t-il. "C'était fou. Je suis d'origine algérienne, et je me retrouvais dans un monde où on faisait comme si je n'étais pas Arabe!" Dafri a alors 27 ans. 
Serein et sans illusions, en apparence, il se dit très content de ce qui lui arrive, mais, ajoute-t-il, "je ne me paluche pas avec ça. Ce qui m'intéresse c'est mon prochain film".

Dans Le Nouvel Observateur du 20 août, il se montre plus acide et peut-être injuste :

"Je suis Français, d'origine algérienne, né à Marseille. Contrairement aux Français de souche, je n'ai ni le complexe du collabo ni celui du colonisateur. Dire qu'il a fallu que ce soit un tortionnaire comme Aussaresses qui parle de la torture en Algérie. Et le cinéma? Il est où notre Platoon sur la guerre d'Algérie? Indigènes, c'est de la merde. Cinématographiquement, c'est pourri. Et puis c'est un film de collabo. Les Arabes, on leur avait promis l'indépendance, en échange de leur mobilisation. Le 8 mai 1945, en fait de libération, les Français les ont massacrés à Sétif. Quand j'ai vu Bouchareb [le réalisateur d'Indigènes] et toute la bande chanter à Cannes 'Nous venons des colonies / Pour sauver la patrie', c'est comme si je voyais des Juifs rescapés des camps chanter 'Heili Heilo"'.

Il faut noter que justement Bouchareb s'apprête à faire un film autour du 8 mai 1945.

Il est plus convaincant quand il explique :

“J'ai 45 ans. Dans ma génération, les mecs des quartiers n'étaient pas aussi retardés que ceux d'aujourd'hui. Ceux-là, on s'est arrangé pour les rendre cons. Ça a commencé avec les socialistes et le paternalisme de Touche pas à mon Pote. Tant mieux si le PS morfle maintenant. Aujourd'hui, les mecs des quartiers sont des connards: ils arrivent à trouver 300 euros pour acheter un iPhone mais pas 20 euros pour acheter un bouquin. Moi, je volais des livres au supermarché. Les Misérables, c'est hollywoodien! Victor Hugo a tout inventé. Il a même inventé 'le fugitif' : Jean Valjean, c'est le docteur Kimble !"

Hafsia Herzi, révélée en 2007 par La Graine et le mulet d'Abdellatif Kéchiche, n'entend pas non plus "être cantonnée à l'Arabe de service". Elle joue dans Le Roi de l'évasion, un film d'Alain Guiraudie sorti cet été. À la question de savoir ce qu'elle pense du “débat concernant la présence sur les écrans d'acteurs issus de l'immigration", l'enfant de Manosque répond :

“ Ce débat ne me parle pas. Il faut se battre et travailler, c'est tout. Évidemment qu'on trouvera toujours des préjugés, mais la situation s'est franchement améliorée pour ma génération. Alors les histoires de femmes voilées, ça va comme ça, ce n'est pas pour moi." (Le Monde, 15 juillet)

2 Yasmine Belmadi interprétait, lui, le rôle du fils de Jean-Pierre Bacri dans Adieu Gary de Nassim Amaouche, prix de la Semaine de la critique à Cannes et sorti le 22 juillet. Le jeune acteur de 33 ans s'est tué dans un accident de scooter quelques jours avant la sortie du film! À propos de son film qui se situe au cœur d'une cité ouvrière, le réalisateur déclare dans le journal Libération du 22 juillet :

"Je voulais absolument rompre avec un certain nombre de clichés liés à la banlieue et à l'immigration. Notamment dans la manière de filmer, sans m'interdire de faire de belles images. La banlieue, du moins telle qu'elle est perçue généralement, et au cinéma en particulier, est totalement déconnectée de l'idée d'une cité ouvrière. Pourtant, c'est ce que sont la plupart des banlieues, justement. Tout le folklore qui s'y attache a fini par faire oublier la condition ouvrière."

Dans Le Monde du 22 juillet, à propos du thème de la mixité, Jean-Pierre Bacri tonne :

“C'est l'avenir du monde! Impossible de la bloquer! Il y a une bande de cons, un certain pourcentage de la population, qui adhère à des discours racistes... Ces gens font le mauvais choix ! La société avance, et qu'on le veuille ou non, elle est mixte !"

Et si Nassim Amaouche aime à se fabriquer des mythes, pour Jean-Pierre Bacri, c'est l'inverse : 
"Moi, c'est le contraire ! Les mythes, je les décapite à la batte de base-ball dès qu'ils naissent! Il n'y a que l'humain qui m'importe! La nostalgie, le conformisme, les mythes, j'éradique !"

\section{Libération du 29 juillet :}

"Les douleurs sourdes, lancinantes et leur corollaire, la colère, sont souvent les plus violentes, voire les plus meurtrières. Ainsi en est-il de celles qu'éprouvent actuellement bon nombre de citoyens africains au sud du Sahara face au sort qui leur est réservé dans la plupart des consulats français du continent. Obtenir un visa pour venir en France n'était déjà pas une sinécure, c'est de plus en plus mission impossible; et aucune catégorie de populations n'est épargnée. Si l'on peut comprendre les nécessités d'une gestion rationnelle des flux migratoires, dans un environnement marqué par de multiples crises, l'on peut néanmoins s'interroger sur la mise en œuvre et les conditions d'application de la politique française d'immigration."

"Chaque jour, les refus de visas se multiplient, notamment en période estivale, où les invitations à participer à des festivals et autres manifestations culturelles sont nombreuses. Pis, les procédés employés pour signifier ces refus sont de plus en plus pernicieux ; les rendez-vous proposés unilatéralement par les consulats sont fixés de telle sorte qu'il est impossible d'honorer les invitations." Et le journal d'égrener : "Invité au festival Africajarc qui se tenait du 23 au 26 juillet à Cajarc dans le Lot, l'écrivain sénégalais Fadel Dia n'a obtenu un rendez-vous que pour le 7 septembre. Les Ivoiriennes Awa et Maaté du groupe Les Go de Koteba ou encore l'écrivain mauritanien Mbarek ould Beyrouck ont eux aussi été victimes de la même logique absurde."

"Si les artistes et les intellectuels qui vivent en Afrique, parfois au péril de leur vie, sont réduits au silence à cause d'une gestion aveugle de l'immigration, alors quid du dialogue des cultures et de la diversité culturelle?" 


\section{RÉSUMÉS}

L'été s'en est allé. La parenthèse estivale s'est refermée sur quelques semaines de repos où les plus chanceux ont pu élargir, un temps, leurs horizons géographiques ou culturels. Pour les autres, le long fleuve, sinueux et agité, de la vie a continué de les brinquebaler. 